\title{
Ultra-violet footpoints as tracers of coronal magnetic connectivity and restructuring during a solar flare
}

\begin{abstract}
L. Fletcher
Department of Physics and Astronomy, University of Glasgow, Glasgow G12 8QQ, UK

e-mail: lyndsay@astro.gla.ac.uk

Received 29 May 2007 / Accepted 16 October 2008

ABSTRACT

Context. The bright, compact ultraviolet sources that appear in flare ribbons are interpreted as sites of energisation of the chromosphere, most likely by electron beams from the corona. Previously we have developed an algorithm to track these compact sources in observations by the Transition Region and Coronal Explorer (TRACE), recording position and intensity. We now exploit this further. Aims. We aim at identifying conjugate footpoint pairs by cross-correlating the TRACE $1600 \AA$ lightcurves in one particular event - the 2002-July-17 M 8.5 flare. We also seek the spatial relationship between the magnetic flux transfer (reconnection) rate, well-connected locations, and energy input by electrons.

Methods. We performed wavelet à trous filtering on the UV light curves, followed by a linear cross-correlation, to identify wellcorrelated pairs. We used RHESSI data to determine the locations of strong electron beam input.

Results. Maps of footpoint pairs were produced in which we can identify well-separated locations that have well-correlated $1600 \AA$ light curves. The time lag between credible conjugate footpoint brightenings can be a few seconds. The flare magnetic connectivity is found to evolve with time. RHESSI hard X-ray sources are found where the flux transfer rate is highest.

Conclusions. We propose that the correlated footpoints are in fact conjugate pairs that are magnetically linked. In some instances, this linkage may be via a coronal null. The time lag in many cases is consistent with excitation by relativistic particles, but correlations with a longer time lag may suggest excitation by waves.
\end{abstract}

Key words. Sun: activity - Sun: flares - Sun: magnetic fields - Sun: UV radiation

\section{Introduction}

During the impulsive phase of solar flares, bright sources of chromospheric emission identify the locations at which excitation of the lower atmosphere is occurring. This excitation is thought to be due to particle beams accelerated in the corona during the flare and travelling to the footpoints. This is argued primarily on the basis that multiple hard X-ray (HXR) footpoints brighten almost simultaneously, implying a rapidly-propagating signal - e.g. an electron beam, and that HXR time profiles at different energies are also consistent with time-of-flight effects on an electron distribution (Aschwanden et al. 1995), although this could also conceivably be an effect of the accelerator.

Flare footpoints are observed to move as the flare proceeds; this is seen in many wavelengths from white-light (e.g. Metcalf et al. 2003) to hard X-ray (e.g. Krucker et al. 2003; Fletcher \& Hudson 2002). This motion is of course not a material motion, but shows the changing location of lower-atmosphere excitation. It reflects the fact that as the flare proceeds, particles are accelerated onto a sequence of magnetic field lines - usually taken as an indication that magnetic reconnection lies at the heart of the energy release process.

In a previous paper, Fletcher et al. (2004) demonstrated an algorithm for tracking impulsive phase UV footpoints. This enables a moving source to be followed through its peregrinations in the lower atmosphere, its position and intensity being recorded as a function of time. In that paper, this information was used to identify correlations between the intensity of the UV flare emission in the $1600 \AA$ channel $\left(I_{1600}\right)$ of the transition region and coronal explorer (TRACE) telescope with the local, instantaneous value of the product $\dot{\Phi}=\dot{x} B_{\mathrm{LOS}}(x)$. This product is measured using the line-of-sight magnetic field found by carefully co-aligning TRACE and magnetograms from the SOHO Michelson Doppler imager (MDI), and the deprojected footpoint "speed". $\dot{\Phi}$ is strictly speaking the flux transfer rate but is often used as a measure of the instantaneous reconnection rate associated with that footpoint. The possibility of determining an "empirical" magnetic connectivity by identifying conjugate footpoints using correlations between the $1600 \AA$ lightcurves from different footpoints was also discussed, and it is this that we attempt here.

If it can be assumed that the time profile of $I_{1600}$ reflects the time-profile of some relevant parameter of the accelerated electrons (most likely the total energy flux per unit area), then footpoint pairs with highly-correlated lightcurves identify magnetic field lines along which highly-correlated particle beams are travelling. It is then reasonable to propose that these pairs are linked to the same unknown particle accelerator or energy release site, so that the magnetic connections in the flare active region, at the time of particle acceleration, can be inferred empirically.

The question of why hard X-ray (HXR) sources are only seen at certain locations along UV flare ribbons can also be addressed with these data. The value of $\dot{\Phi}$ is presumed to be related to the rate at which magnetic energy is released, so we will investigate in detail the spatial relationship between $\dot{\Phi}$ and HXR source location.

In Sect. 2 we briefly summarise the flare event, and the work of Fletcher et al. (2004), to set the context. Section 3 describes 
the preparation of the data and the filtering used, and Sect. 4 presents the correlations found. The relationship between flux transfer rate and HXR source location is shown in Sect. 5 and we end with discussion and conclusions in Sect. 6.

\section{Summary of the event and previous work}

The flare under consideration took place in active region complex, NOAA region 00030, at the interface between an older decaying spot pair to the west and a newer pair to the east. This region produced a series of $\mathrm{M}$ and $\mathrm{X}$-class events in the period 9-18 July 2002. The 2002-July-17 M 8.5 flare which we study here had two UV bursts. The first burst peaked in $1600 \AA$ from 07:02 to 07:03 UT, and the second peaked around 07:12. We analyse the first of these two bursts. (One of the other events from this region, on 2002-July-15 X3.0 has been the subject of other studies, e.g. Gary \& Moore 2004; Harra et al. 2005, and we note that it would also be amenable to the analysis that we present.)

The main flaring activity on 2002-July-17 occurs around the neutral line between the new active region's positive flux, and the old active region's negative flux. There are also UV footpoints present in the positive field of the older region, and early in the flare there are small UV flashes in its negative field. However, when TRACE goes into flare mode, this location is unfortunately outside the TRACE field of view.

In Fletcher et al. (2004) we described an algorithm developed to track small-scale moving features in solar images. This algorithm was applied to a sequence of TRACE $1600 \AA$ images of an M 8.5 flare that occurred on 2002-July-17. Over the course of the flare, around 120 individual footpoint sources within the overall UV ribbon were identified and tracked. The sources showed an overall "drift" associated with the separation of flare ribbons as the flare proceeded, with a superposed "diffusion", consistent with the footpoints meandering around the boundaries of granular cells. Footpoints were observed to move rapidly into the penumbral region of a sunspot, following the direction of bright penumbral filaments.

For each footpoint individually, the strength of emission in the $1600 \AA$ channel of TRACE was recorded as a function of time, and compared with the value of $\dot{\Phi}$. It was found that peaks in the intensity of $1600 \AA$ emission are significantly correlated in time with peaks in the product $\dot{\Phi}$. In other words, on the scale of individual footpoints, there is a suggestion that the energy input fluctuates with the local reconnection rate.

\section{Data analysis}

A series of 139 TRACE $1600 \AA$ images, at a cadence of $2 \mathrm{~s}$ and beginning at 2002-Jul.-17 07:01:31 was used in this analysis. The data were prepared by subtracting the detector pedestal of $86 \mathrm{DN}$, normalising by the UV flat-field, and then by exposure time. The images were co-registered by obtaining a value for pointing drift and jitter through cross-correlation on a patch of network. Individual bright sources on these co-registered and calibrated flare images were then tracked using the tracking algorithm mentioned above. Full details of the data reduction and tracking, along with samples of the UV intensity profiles, can be found in Fletcher et al. (2004).

$I_{1600}$ flare time profiles exhibit both rapidly varying - i.e. bursty $(\sim 10 \mathrm{~s})$ and slowly-varying components. The peaks of the $I_{1600}$ lightcurve summed over all sources correlate well in time with hard X-ray emission, to within instrument and counting

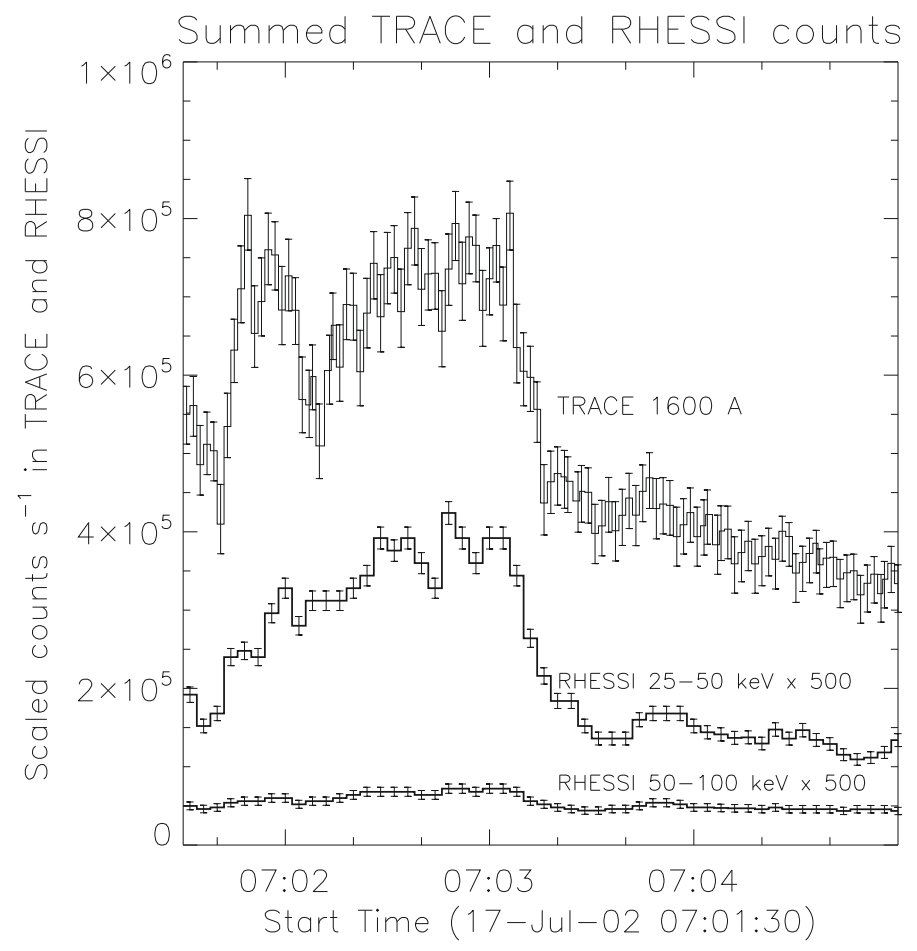

Fig. 1. The UV and HXR lightcurves from the 2002-Jul.-12 M 8.5 flare. The UV curve has been obtained by summing all emission over an area of $75^{\prime \prime} \times 35^{\prime \prime}$, centred on the flare, and normalising to the shortest exposure time in the sequence, $0.0064 \mathrm{~s}$. The HXR light curves are RHESSI summary data, corrected for shutter motions. Error bars on the TRACE data are the combined photon counting error and statistical error on the background pixel values. Error bars on the RHESSI datapoints are photon counting error.

limitations (Fig. 1). The interpretation is that the UV emission occurs during intervals of rapid heating of the lower atmosphere by fast particles.

We wish to separate out the bursty components, assuming that these are caused by discrete injections of electrons. The more slowly varying component may be a gradual thermal response. Aschwanden et al. (1995) in their analysis of BATSE HXR data, used a Fourier decomposition, subtracting off the most slowly-varying Fourier components to emphasise the more rapidly-varying terms. This of course forces the time series to be decomposed into sums of sines and cosines, which may not be the best method for aperiodic bursty signals. Many previous authors have introduced wavelet transforms into solar physics, as a means for decomposing time series to extract quasi-periodic signals often having short duration and periods that change during the observation interval (e.g. De Moortel et al. 2000; King et al. 2003; De Pontieu et al. 2003). In general, a wavelet transform is the convolution of the data with a set of wavelet functions (based upon a so-called "mother wavelet"), with properties such as number of oscillations or characteristic time scale covered by the wavelet that are in some sense attuned to the data analysis problem in hand. The output of such wavelet analyses is usually presented as a 2-D power spectrum in wave period and in time. Ours is a rather different problem, in which we have no expectation of periodicity (or lack of it) but wish to separate out features in the light-curve on the basis of a few characteristic timescales alone. In this work, we use the à trous wavelet transform (Starck et al. 1997; Starck \& Murtagh 2002), which decomposes the time series into features on only a few separate and independent scales (durations), rather than providing 

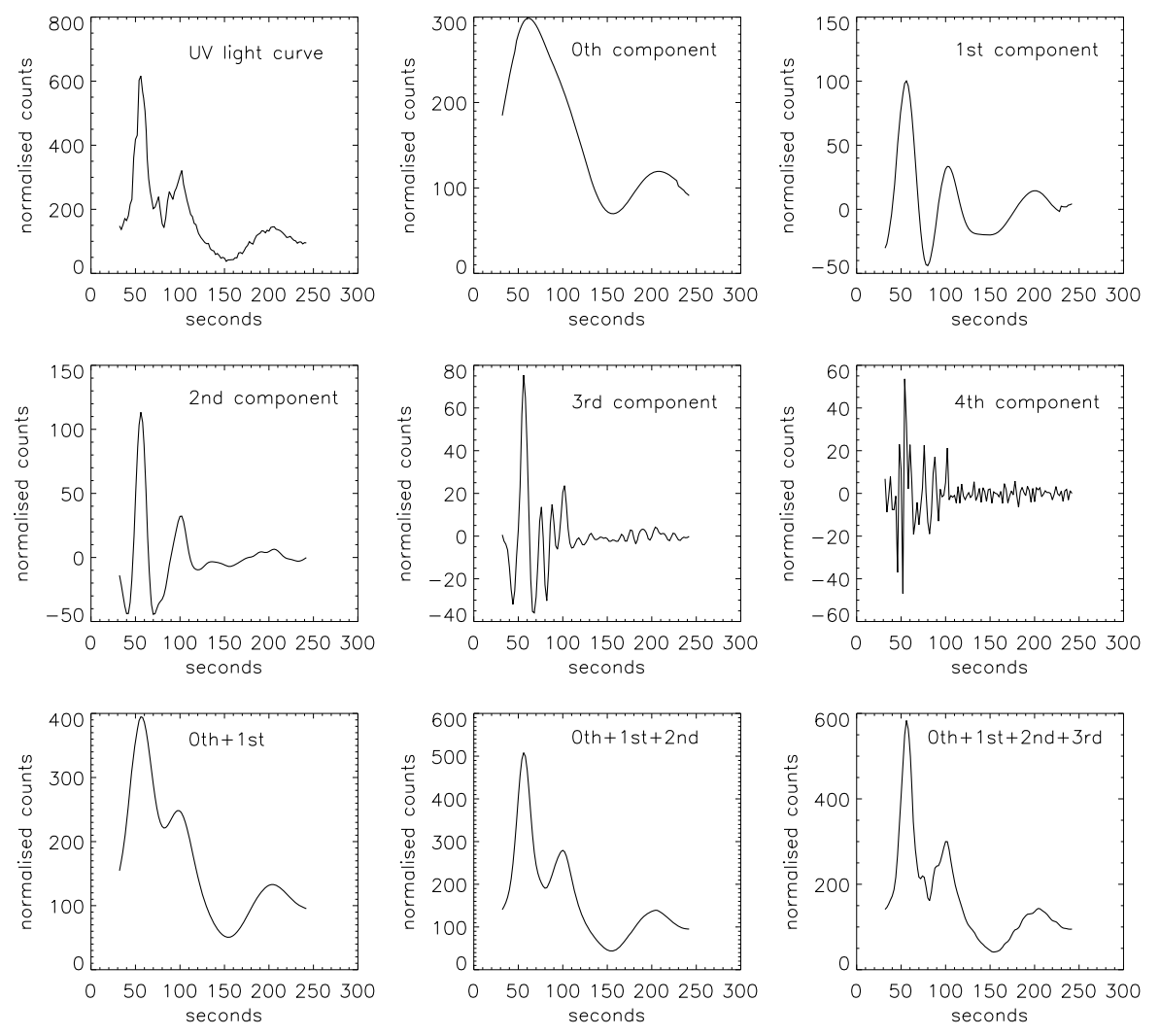

Fig. 2. The UV lightcurve of a single UV footpoint broken down into its wavelet components using the à trous algorithm.

power on all scales as in the usual wavelet periodogram. Apart from being fast and straightforward to implement (being carried out in real space rather than having to transform the data), the à trous decomposition allows one to readily distinguish the different scales of interest, with the original data being trivially reconstructed as the sum of the components. Likewise, it becomes easy to identify and subtract off a slowly-varying background, or high frequency noise. The wavelet we use is the $B \_3$ spline described in Starck \& Murtagh (2002) and we examine structure on 5 timescales starting at the Nyquist limit (twice the smallest time interval, or $4 \mathrm{~s}$ ), and with each timescale differing from the previous by a factor of two.

An example of the decomposition is shown for a typical $I_{1600}$ lightcurve in Fig. 2. It can be seen that burst scales of about 10-20 s FWHM, corresponding to the "elementary flare bursts" described by (e.g. Grigis \& Benz 2005), are well-detected by the 2nd and 3rd wavelet components. Scale 4 contains (as well as possible real structure) high frequency variations caused mainly by differences in the very short exposure times required for these bright UV sources, which we suspect are not measured accurately by the instrument and cannot therefore be corrected for accurately. The zeroth and first components contain the slow variations. Although we have reason to filter out the slowest and the most rapid variations, after that there is no reason a priori to neglect any of the other scales. Therefore, in carrying out the analysis in this paper, we will filter the data to retain different sets of components, comparing results retaining components [1, 2, 3] and $[2,3]$; Fig. 3 shows an example of these.

For comparison and illustration, we show sample results of the usual Fourier filtering procedure, subtracting low and high frequencies from the signal, leaving only intermediate scales. Figure 4 shows the result of Fourier filtering the same lightcurve as is used in Fig. 2, to isolate frequency bands separated by factors of two, similar to the à trous separation by timescale.
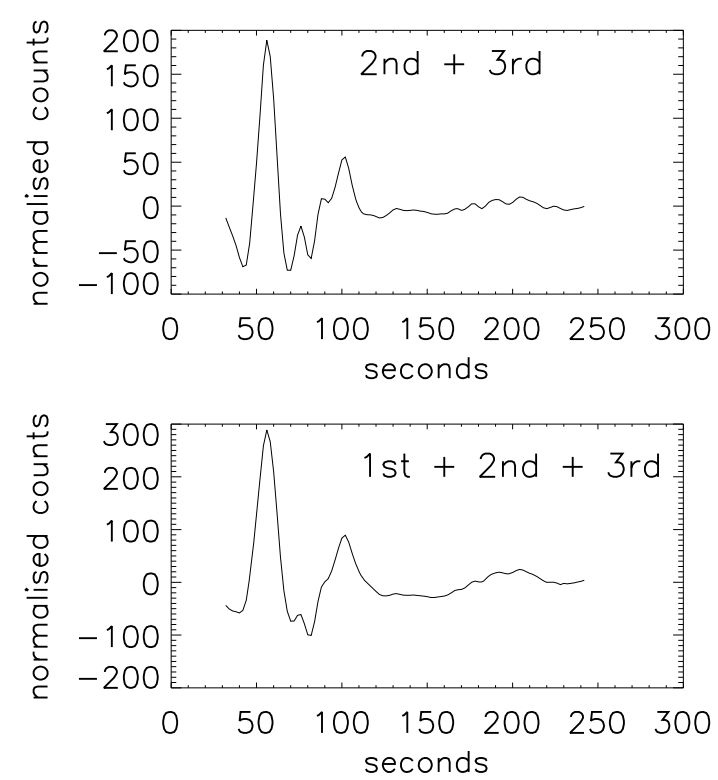

Fig. 3. The shorter timescale components of the UV lightcurve shown in Fig. 2. These emphasise variability on timescales of around $10 \mathrm{~s}$.

Results are similar to those obtained using the à trous decomposition but because the Fourier transform uses sines and cosines of infinite extent, periodic signals are present in these filtered signals (e.g. beyond $\sim 150 \mathrm{~s}$ ) which do not in isolation represent the lightcurve behaviour in this location - they are only removed by adding back in the other frequencies. Correlation on such Fourier-filtered signals would give a poor result.

UV sources come and go throughout the flare, and we will focus initially on those sources which are present in the first $120 \mathrm{~s}$ of the event, from 07:01:30 UT to 07:03:30 UT. Figure 6 

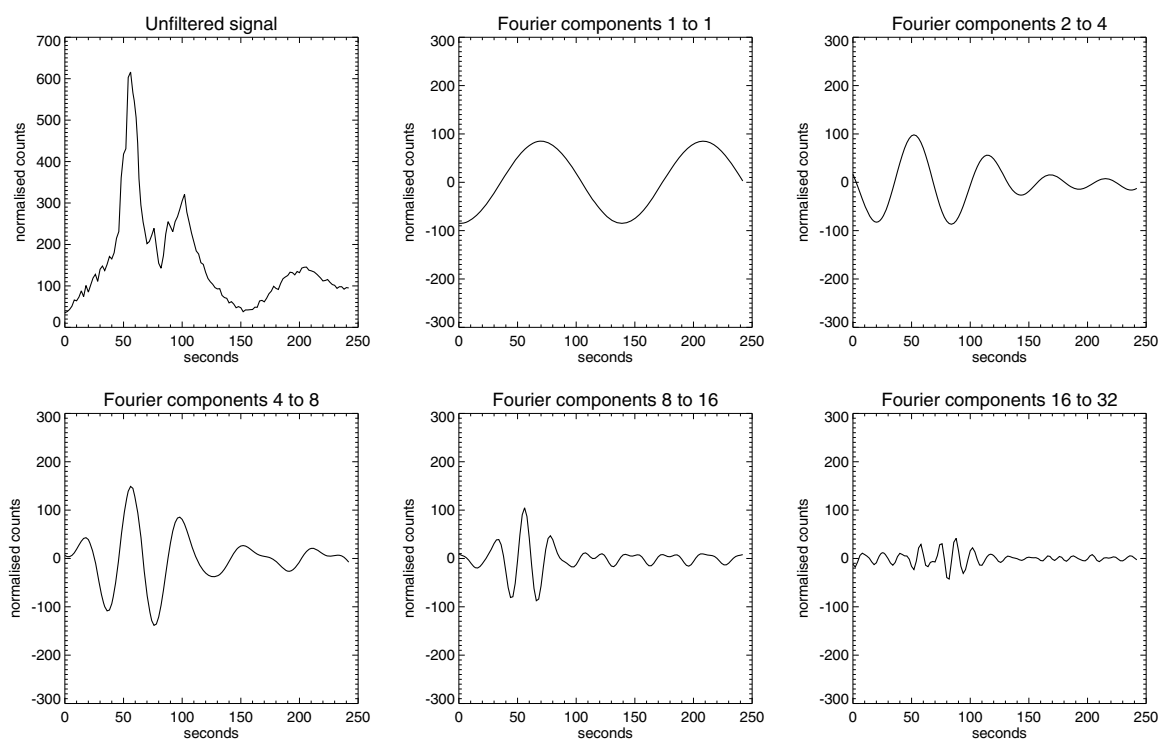

Fig. 4. The UV lightcurve of a single UV footpoint filtered using standard Fourier techniques, to isolate features with frequencies separated by factors 2 . Fourier analysis is not used in this paper, but the plot allows one to compare the standard Fourier decomposition with the $\grave{a}$ trous decomposition shown in Fig. 2.

shows the unfiltered, stacked lightcurves for the first $120 \mathrm{~s}$, each of which has been normalised to the largest value in that lightcurve, then added to a constant offset. The numbers on the right hand side of the plot refer to the (somewhat arbitrary) source identifier, which can be located using the map in Fig. 5. By inspection, different sources vary in more or less coordinated ways with their neighbours, and also with more distant sources. The peaks in the lightcurves are apparently not just random brightenings; there are patterns of excitation visible. Between neighbouring sources, there is the possibility of cross-talk between sources due to the TRACE point spread function which has a $F W H M$ of about 3 TRACE pixels. For distinct sources separated by more than twice the FWHM this is expected to be minimal. For example, well-separated source pair $[4,49]$, or $[113,58]$ have significant peaks at the same times. Removing à trous component 4 (i.e. the high-frequency noise) results in the light curves shown in Fig. 7.

\section{Correlated intensity variations and implications for magnetic connectivity}

The à trous-filtering generates edge effects, therefore we crop the filtered lightcurve at the start by $30 \mathrm{~s}$ before correlating. The filtered and cropped lightcurve of every footpoint present in the first $120 \mathrm{~s}$ of the event is then correlated against that of every other footpoint using the Pearson linear correlation coefficient as a measure, and admitting a variable time lag between curves. Assuming that the UV footpoints are excited by fast electrons, the time lag between correlated footpoints ought to be less than a second (i.e. a typical loop length of $10^{9}-10^{10} \mathrm{~cm}$ traversed at around 0.4 of the speed of light for a $50 \mathrm{keV}$ electron). However, out of curiosity we allow the time lag to be larger than this.

There are 59 such footpoints, so $\sim 1700$ possible pairings, and it is obviously not practical to examine each one. Therefore, a threshold for a good correlation is set corresponding to a Pearson correlation coefficient of greater than 0.8. Among this set, many pairs of correlated close neighbour footpoints are found which are not interesting from the point of view of examining large-scale connectivity, although it can provide information about coherent scales in the excitation. We select out only those correlated pairs which are separated by more than 20

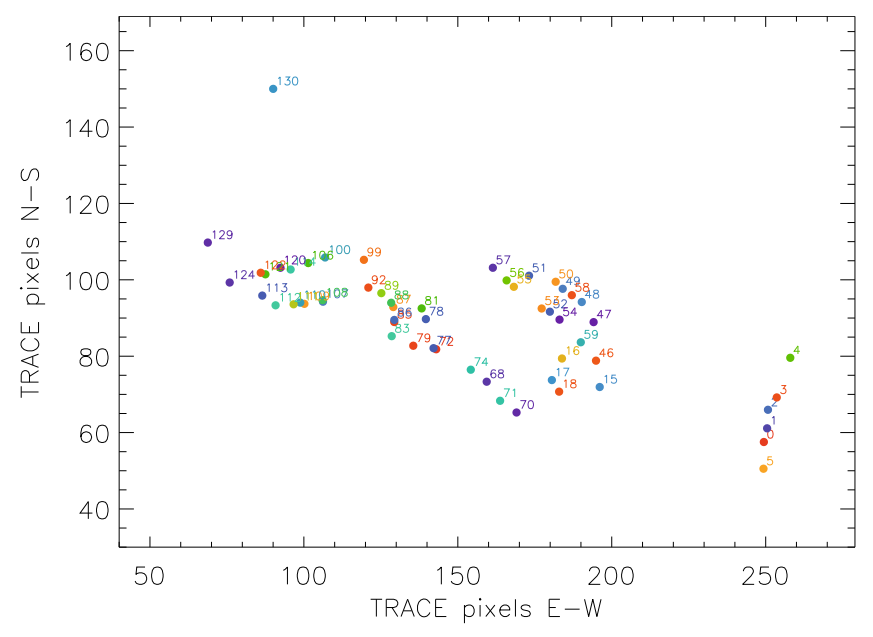

Fig. 5. Source identifiers for the sources present during the first $120 \mathrm{~s}$ of the flare.

TRACE pixels, or 10" (this also avoids the problem of source cross-talk, as described in Sect. 3).

The pairs recorded as "strongly correlated" depend to some extent on the number of wavelet components used in obtaining the correlation. To give an idea of the quality of the correlations, we show examples of the correlations obtained using components [2,3] and [1, 2, 3], allowing a time lag of less than $8 \mathrm{~s}$. Figure 8 shows the correlations between footpoint pairs that were present at the start of the TRACE time sequence at 07:01:31, obtained using wavelet components [2,3]. Figure 9 is the same but using components $[1,2,3]$. In the corner of each panel is the correlation coefficient as a function of time lag.

The footpoint pairs which show high correlation coefficients are overplotted on a map of the magnetic field. In the upper panel of Fig. 10 the highly-correlated pairs of footpoints (correlation coefficient over 0.8 ) using components [2, 3], and with a time lag less than $5 \mathrm{~s}$, are linked by a semicircle. This is intended only to guide the eye as to conjugate pairs, and does not show the shape of the supposed field. The right-hand panel of this figure shows the correlated pairs found using components $[1,2,3]$. In this panel, there is one loop which joins a positive field sources to another positive field source. This is patently not a field line, however may imply communication via the magnetic 


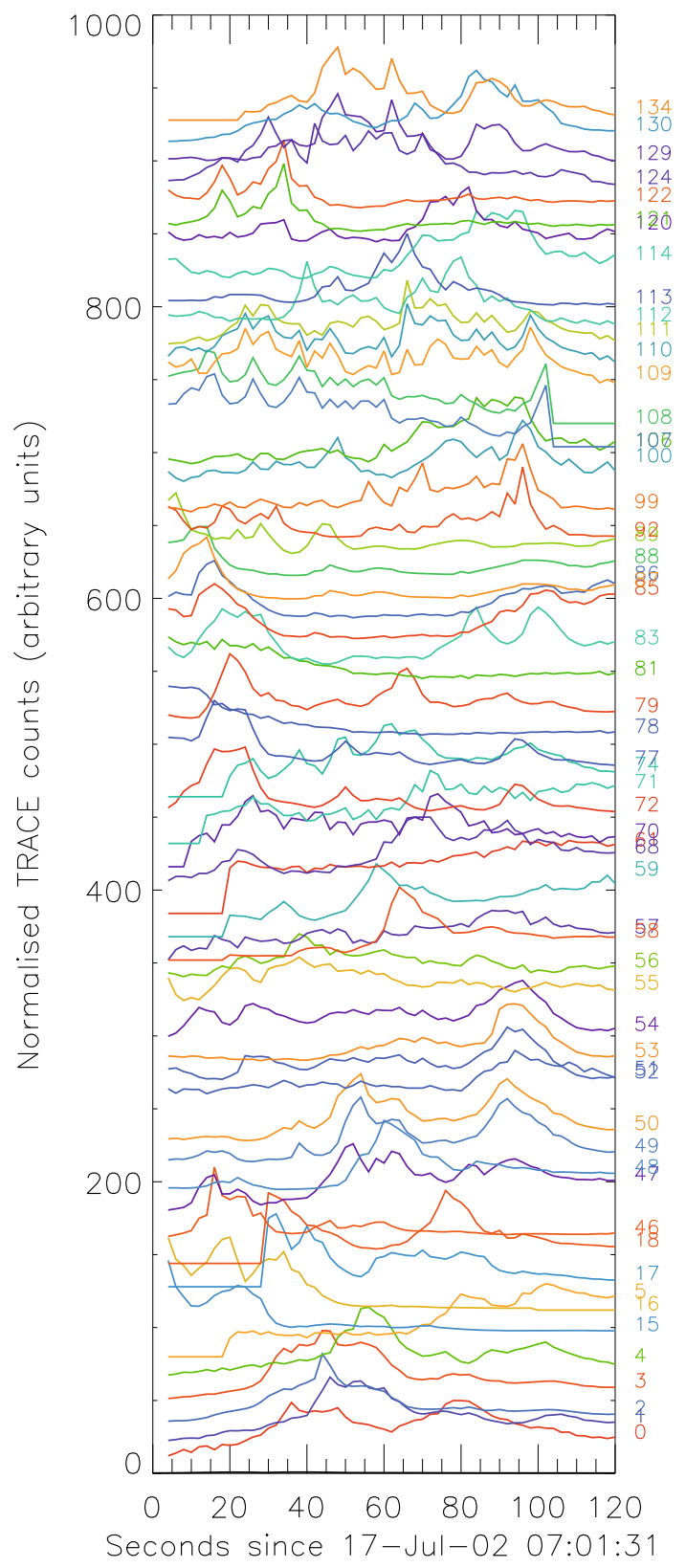

Fig. 6. $1600 \AA$ lightcurves for the individual UV footpoint sources present during the first $120 \mathrm{~s}$ of the flare. The numbers on the right hand side of the plot are the source identifier.

field - for example at a coronal null. It is interesting that a few minutes after the later flare UV burst at 07:12 UT (which we do not analyse here) there are flows visible in $1600 \AA$ suggestive of the presence of a coronal null structure above the region between the negative field and the western positive field. The photospheric distribution of magnetic sources - i.e. a negative patch surrounded by positive patches - is a typical arrangement in which a coronal null might occur. More generally, Gorbachev et al. (1988) and Maclean et al. (2006) find topological states consisting of two bipoles (in this event, the older and the newer bipole comprising the active region complex) with a coronal null, so such a configuration is plausible here.

The most numerous, and robust, connections are found across the neutral line between the main positive and negative field patches in Fig. 10, i.e. the negative polarity of the older region and the positive polarity of the newer region. There are also

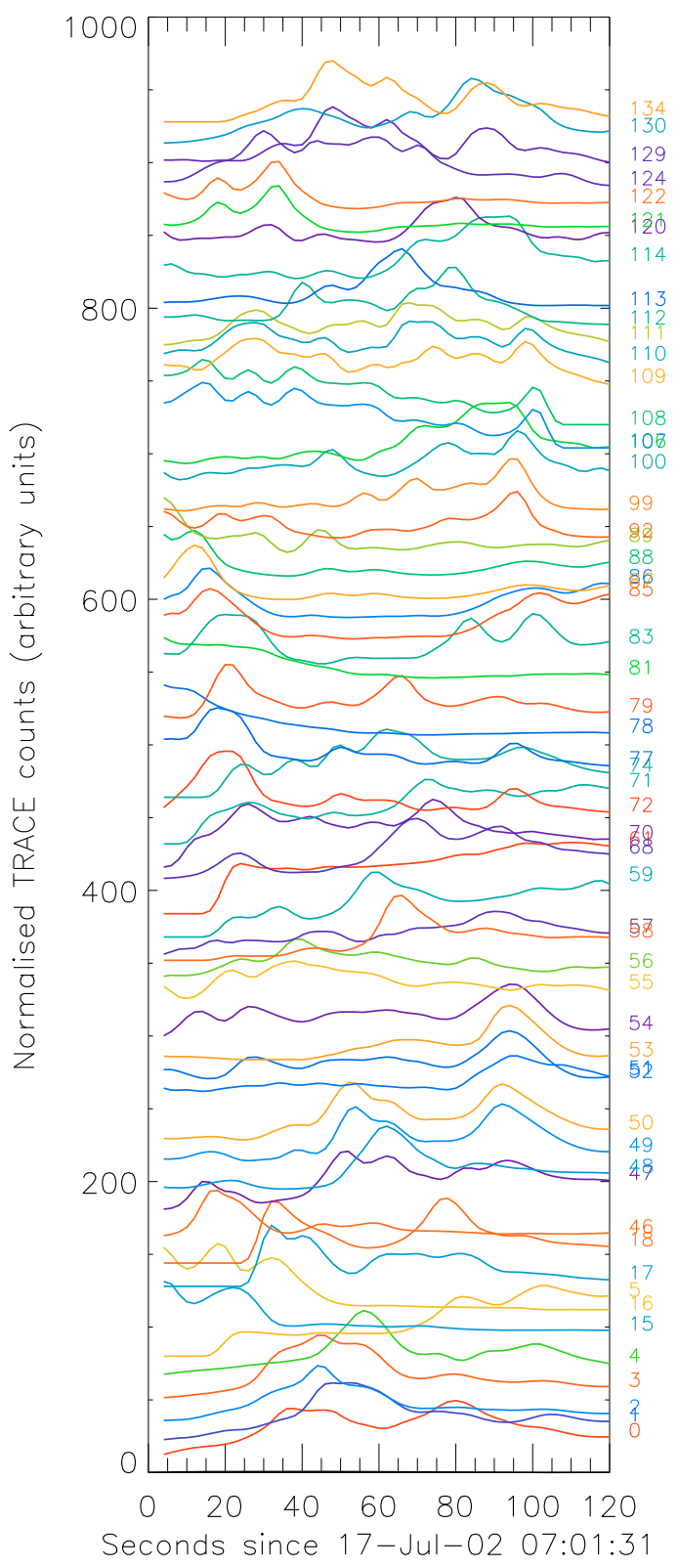

Fig. 7. $1600 \AA$ lightcurves for the individual UV footpoint sources present during the first $120 \mathrm{~s}$ of the flare, filtered with the à trous wavelet to remove the highest frequency noise, i.e. component [4].

correlated pairs across the neutral line within the older (western) region. Under the hypothesis that the footpoint pairs are illuminated as a result of magnetic reconnection, the presence of so many pairs across the inter-group neutral line indicates that connections are being formed between the two spot groups.

If the UV emission is generated by fast particles accelerated in the corona (and noting that the UV and HXR emission is wellsynchronised), one would expect that the time lag between conjugate pairs would be less than $1 \mathrm{~s}$. There are many instances of time lags consistent with this. But there are also instances of well-correlated footpoints with time lags on the order of a few seconds. It is not clear what this means. Firstly these "correlations" could simply be chance alignments. Secondly, each footpoint has different initial plasma conditions and the shift in time could reflect the different times taken for the chromosphere in different locations to reach the conditions for UV emission when bombarded by a beam (which might, for example, be 

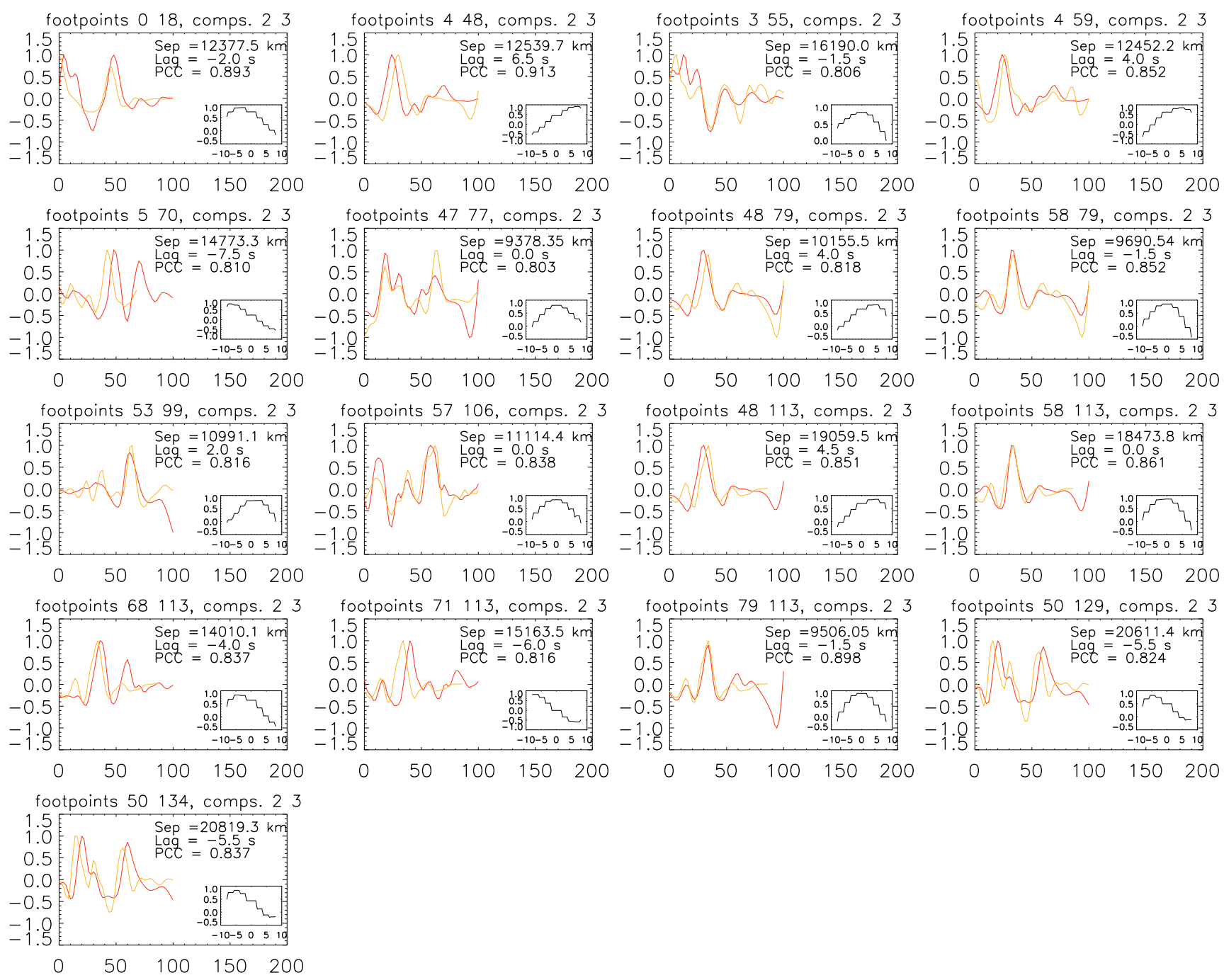

Fig. 8. Lightcurves of footpoint pairs found by the algorithm to be highly-correlated when using wavelet components [2, 3]. In the plots, the lightercoloured lightcurves have been shifted relative to the darker lightcurved by the time interval which gives the highest Pearson linear correlation coefficient. The $x$-axis is time in seconds since 17-Jul.-02 07:02:03. The inset in each plot is the correlation coefficient as a function of lag.

weak in one footpoint and strong in another). Thirdly, the UV footpoints could be excited not by particles but by some other mechanism such as Alfvén waves with a slower coronal travel speed. This has been discussed from a theoretical point of view by Emslie \& Sturrock (1982), Melrose (1992) and Fletcher \& Hudson (2008), who proposed MHD waves as a means of transporting flare energy from corona to chromosphere. Time lags of say $2 \mathrm{~s}$, over loop-lengths of order 50 to 100 TRACE pixels (18000 to $36000 \mathrm{~km}$ ) would correspond to high wave speeds of up to tens of thousands of kilometers per second. In a sufficiently strong coronal field in a low density corona (say $300 \mathrm{G}$ at an electron number density of $<10^{9} \mathrm{~cm}^{-3}$ ) this would be achievable.

The only previous attempt to carry out footpoint pair time correlations was made by Sakao et al. (1994), using Yohkoh Hard $\mathrm{X}$-ray Telescope data. They found time lags consistent with zero, except for one case where a time lag of $1.5 \mathrm{~s}$ was found. It is possible that different radiation types is excited in different ways. However, it should also be realised that the imaging algorithm used in that study (the Maximum Entropy Method) tends to redistribute counts from stronger to weaker sources, leading again to cross-talk. Therefore their zero-lag correlations might be partly artefactual.

As can be seen from Figs. 8 and 9, the correlation is not consistently good throughout the lightcurves; pairs which are initially well-correlated may lose that correlation over the correlation window of $120 \mathrm{~s}$. This loss of correlation may be due to changing connections at the reconnection site, or at the footpoints, but it is difficult to distinguish between the two on the basis of these data. The characteristic timescale associated with changing connections, i.e. that on which new field is advected into the reconnection region, would be on the order of the size of the active region $(30000 \mathrm{~km})$ divided by the Alfvén speed $\left(1000 \mathrm{~km} \mathrm{~s}^{-1}\right)$ or some tens of seconds. This is comparable to the variation timescale one would expect for flare heating-induced changes to chromospheric conditions in a stationary footpoint, i.e. the evaporation timescale, approximated by the sound-travel time vertically through the chromosphere, or $1000 \mathrm{~km} / 10 \mathrm{~km} \mathrm{~s}^{-1}=100 \mathrm{~s}$. If the footpoint moved to a part of the chromosphere with different conditions, UV intensity changes on the "self-crossing" time for the footpoint would be expected (about 4 TRACE pixels at $/ 15 \mathrm{~km} \mathrm{~s}^{-1}$, or about $200 \mathrm{~s}$ ). This would lead to random correlated pairs rather than 


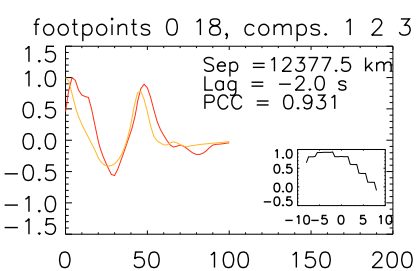

footpoints 355 , comps. 123

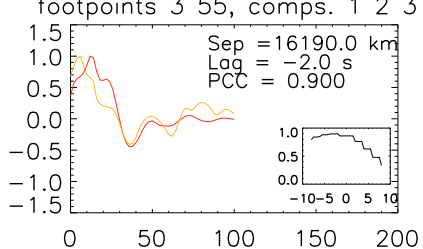

footpoints 5879 , comps. 123

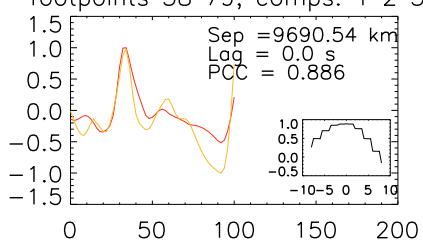

footpoints 5285 , comps. 123

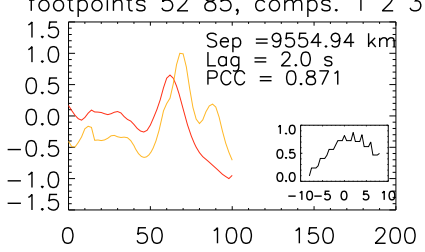

footpoints 5386 , comps. 123

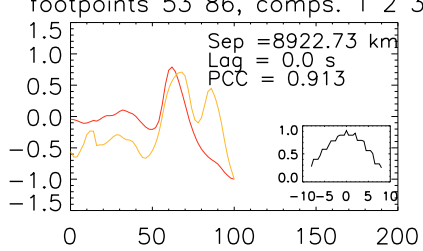

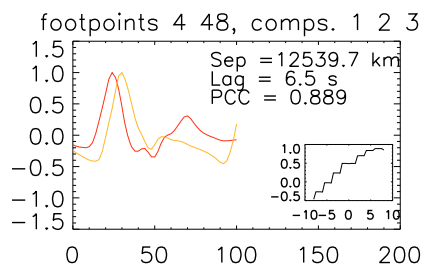
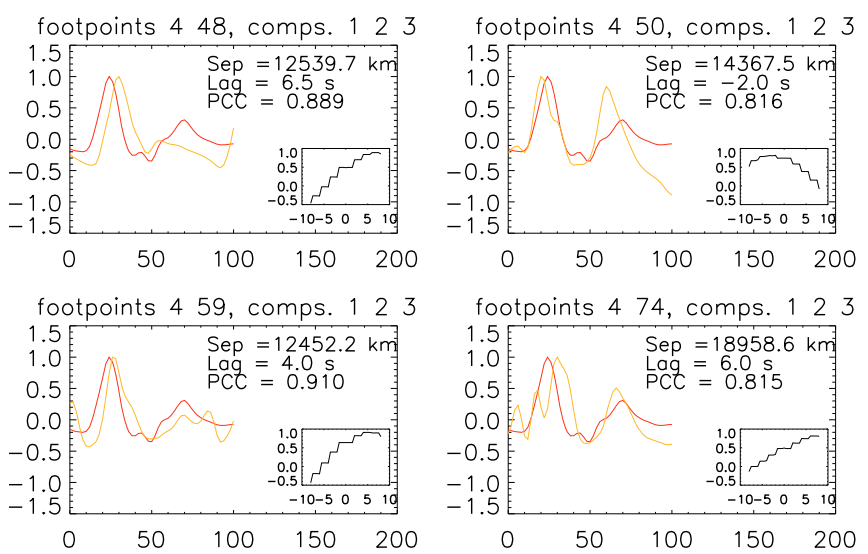

footpoints 474 , comps. 123

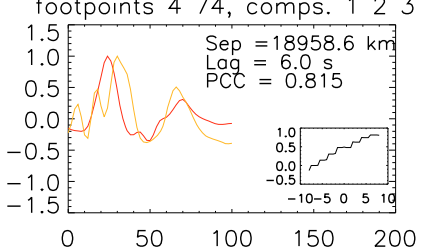

footpoints 1781 , comps. 123
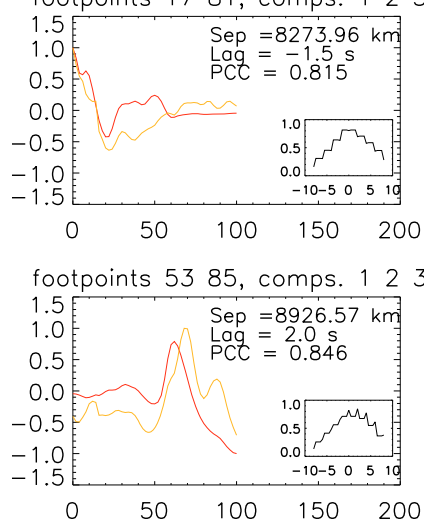

footpoints 5486 , comps. 123

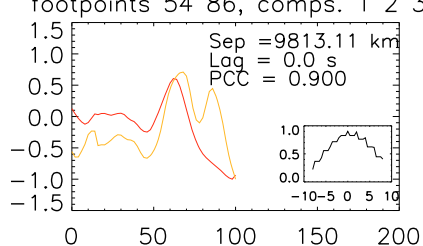

footpoints 6181 , comps. 123

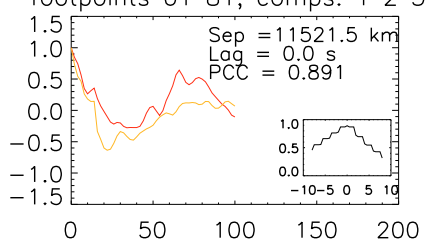

footpoints 5485 , comps. 123

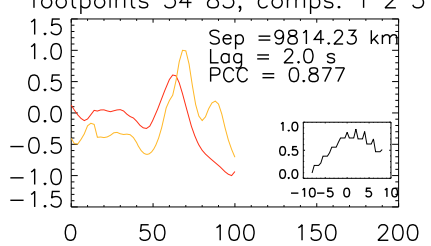

footpoints 5399 , comps. 123

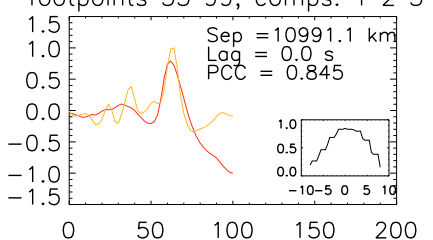

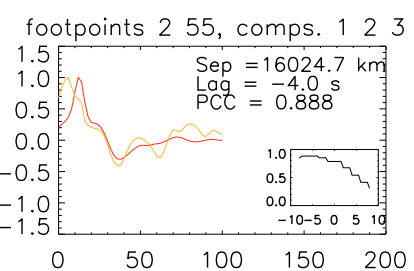

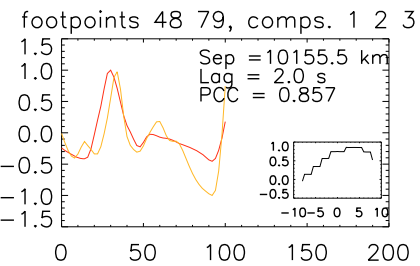

footpoints 5185 , comps. 123

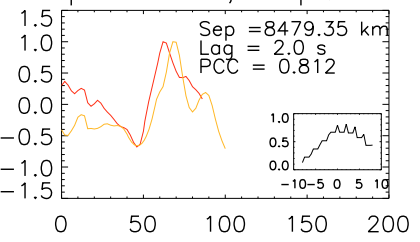

footpoints 5286 , comps. 123

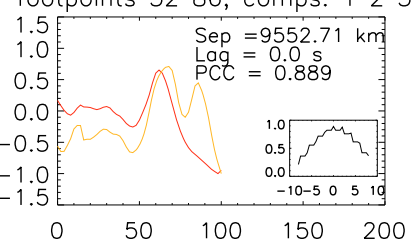

footpoints 57100 , comps. 123

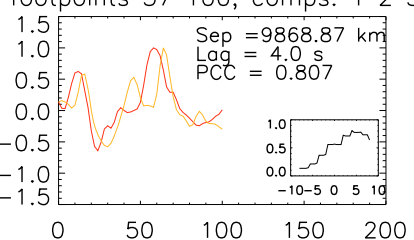

Fig. 9. As Fig. 8, but correlating now on wavelet components $[1,2,3]$. Only the first 20 of 30 correlated pairs are shown.

the predominantly positive-negative footpoint pairs seen, so we discount this possibility.

However, under the assumption that the evolving correlations are due to changing magnetic connectivities, we examine the connectivities implied at a later time. In Fig. 11 we show the correlated footpoint pairs present in a different but overlapping time interval between 07:02:30 and 07:04:08. Because more footpoints are illuminated at this time than in the earlier period, the number of actual and randomly correlated pairs is higher, so that using the same lower cut-off of correlation coefficient $(0.8)$ as is used in Fig. 10 would result in a crowded plot. Therefore, to simplify the figure, the critical value of the correlation coefficient for plotted pairs is set higher, at 0.9. It is clear that the connections have changed compared to Fig. 10. In particular there are an increased number of long connections joining positive field to positive field. So although the main neutral line is still inferred to be the primary site of reconnection, it may be that the null becomes more involved as the flare proceeds, and field at ever higher altitudes reconfigures.

\section{Relationship of UV footpoint motions to particle acceleration}

The product of the footpoint apparent speed and the line-of-sight magnetic field, expressed as a flux transfer rate $\dot{\Phi}$, has been taken by many authors, (e.g. Qiu et al. 2002; Fletcher et al. 2004; Temmer et al. 2007), as a measure of the coronal reconnection rate. It should be stressed that such ideas were motivated initially by observations of the gradual phase of large two-ribbon flares (Kopp \& Pneuman 1976), where the magnetic configuration is well-approximated by a 2- or 2.5-D field, and in which flare ribbons move steadily outwards and away from the magnetic neutral lines. In such configurations, the coronal reconnection rate is also equal to the coronal reconnection electric field (Poletto \& Kopp 1986). In some models for example the early work of Litvinenko \& Somov (1993), it is this field that is directly responsible for the acceleration of flare particles. Again in a strictly or almost 2-D configuration (i.e. no significant shear or twist component of the field), and also assuming that all energy is dissipated within the reconnection region, the energy release rate is linearly related to the Poynting flux into the current sheet (e.g. Lee et al. 2006), the latter also being determined based on the motions of footpoints, and the line-of-sight magnetic field strength, at the flare footpoints (e.g. Asai et al. 2004; Temmer et al. 2007).

However the impulsive phase of a flare is observationally very complicated and three-dimensional, with the main impulsive phase footpoints often moving parallel or antiparallel to each other and to the magnetic neutral line (Bogachev et al. 2005), and the ribbons overall sometimes remaining relatively 

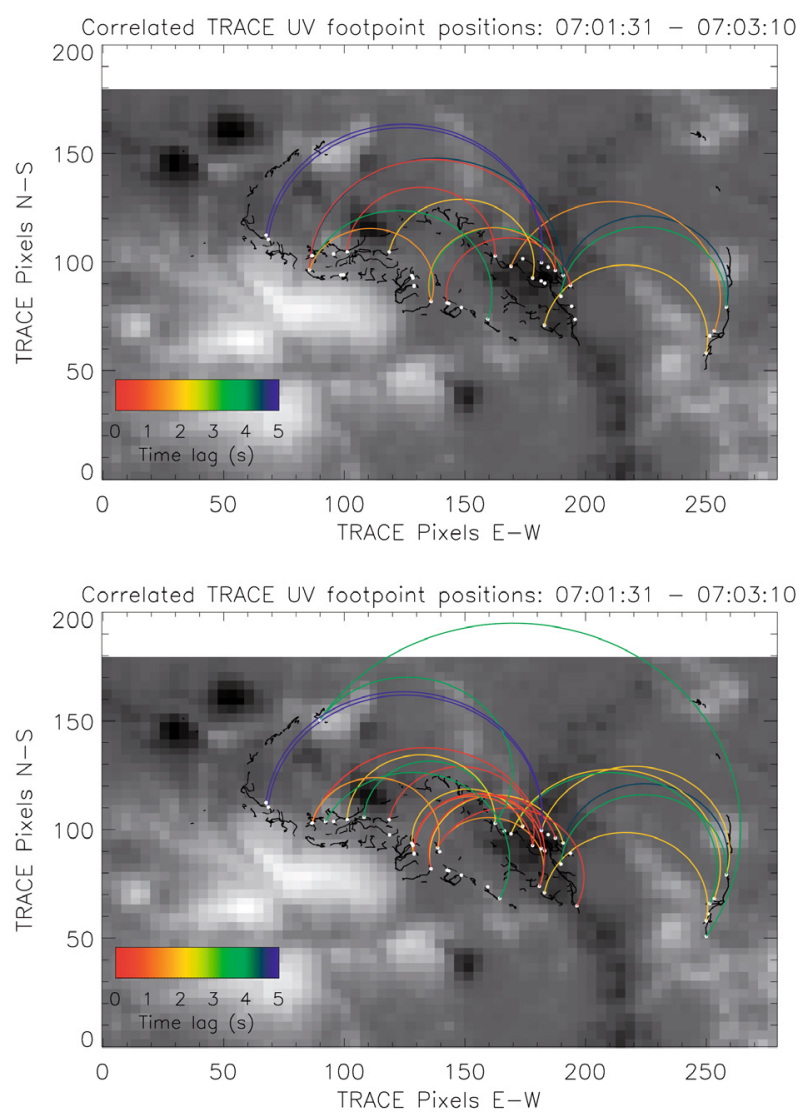

Fig. 10. Pairs of footpoints, colour-coded by time lag between lightcurves, correlated within $5 \mathrm{~s}$ with a correlation coefficient greater than 0.8. Upper: correlations resulting using à trous components [2, 3]. Lower: correlations resulting using à trous components $[1,2,3]$.

stationary (e.g. Saba et al. 2006). Although the relationship between footpoint motion, magnetic field and flux transfer rate is preserved for the flare as a whole (it being a consequence of conservation of magnetic flux) the coronal reconnection electric field is not so readily obtained. Nor, in an environment of strong twist and shear (i.e. high non-potential energy density) will there be a simple relationship between $\dot{\Phi}$, the Poynting flux and the energy release rate. The first investigation of the relationship between reconnection, coronal electric fields and locations of flare ribbons in a 3-D structure was the analysis of Hesse et al. (2005), who derived a relationship between the instantaneous reconnected magnetic flux at a field line and the parallel electric field along that field line, suggesting also that the endpoints of field lines with high values of parallel electric field correspond to locations of chromospheric excitation. The actual relationship between $\dot{\Phi}$ and the parallel field is determined by the coronal magnetic configuration, which could be prescribed by Hesse et al. (2005) but is not generally known.

Nonetheless, this analytic work can give us some confidence that $\dot{\Phi}$ is a meaningful quantity, even for the impulsive phase, and observationally there are interesting correlations between $\dot{\Phi}$ and properties of the footpoint radiation, such as the intensity in TRACE $1600 \AA$ emission (Fletcher et al. 2004; Saba et al. 2006) and the integrated non-thermal hard X-ray flux in sources moving in the direction of the field gradient (Qiu et al. 2002). Since the Ramaty high energy solar spectroscopic imager, RHESSI, (Lin et al. 2002) also observed this flare, we have the opportunity to combine detailed observations of the magnetic
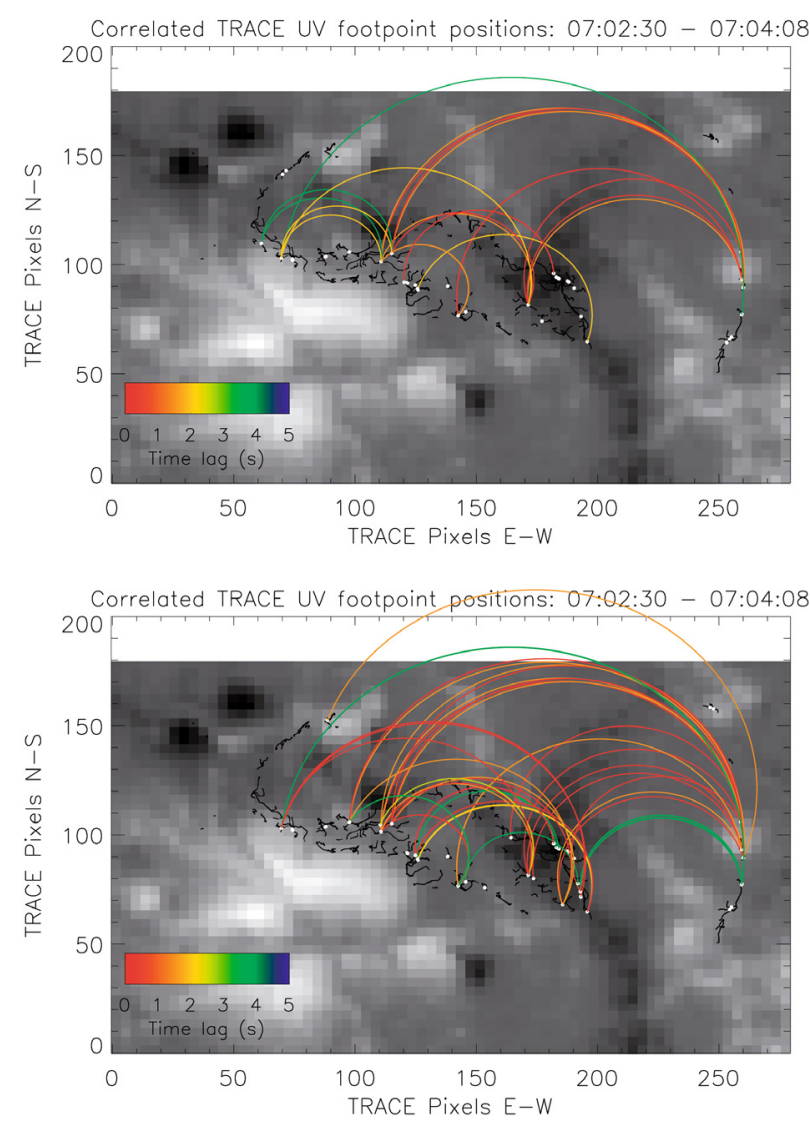

Fig. 11. As Fig. 10 but correlating over a later time interval and showing pairs with a correlation coefficient greater than 0.9 .

flux transfer rate implied by footpoint motions with the sources of HXR emission.

The process of deriving $\dot{\Phi}$ from the UV and magnetic observations is described in Fletcher et al. (2004) and we shall not repeat it here. The rate ranges from around 10 to $10000 \mathrm{~V} \mathrm{~m}^{-1}$. For each footpoint at each time we code the value of this by colour, and in Figs. 12 and 13 we superpose it on an MDI magnetogram and overplot the HXR contours at 25-50 and 50-100 keV, made using the "Pixons" algorithm and RHESSI grids 1, 3, 4 and 6.

These figures show that, in the main, the sites of strong HXR emission are concentrated at locations with a high value of $\dot{\Phi}$ (redder footpoints). This confirms, but in more detail, the findings of Temmer et al. (2007), who carried out a similar analysis on the mean motion of flare $\mathrm{H}_{\alpha}$ ribbons and the relationship to HXRs. We point out one significant new effect. As can be seen from the UV footpoint tracks shown in Fig. 5 of Fletcher et al. (2004), the individual footpoints located in the strongest negative field (at $\sim(190,100)$ in Fig. 12) are converging rapidly, rather than having a significant net "spreading" speed away from the positive polarity footpoints. This rapid convergence leads to a high $\dot{\Phi}$ - of order a few $1000 \mathrm{~V} \mathrm{~m}^{-1}$, whereas if only the average speed of this group of sources were to be measured (i.e. without tracking the individual elements) the resulting $\dot{\Phi}$ would be significantly lower. This highlights the potential errors in trying to analyse ribbon properties as a whole, without accounting for their sub-structure.

\section{Discussion and conclusions}

We have presented here a correlation analysis of individual flare ultraviolet footpoints observed in the TRACE $1600 \AA$ channel. 

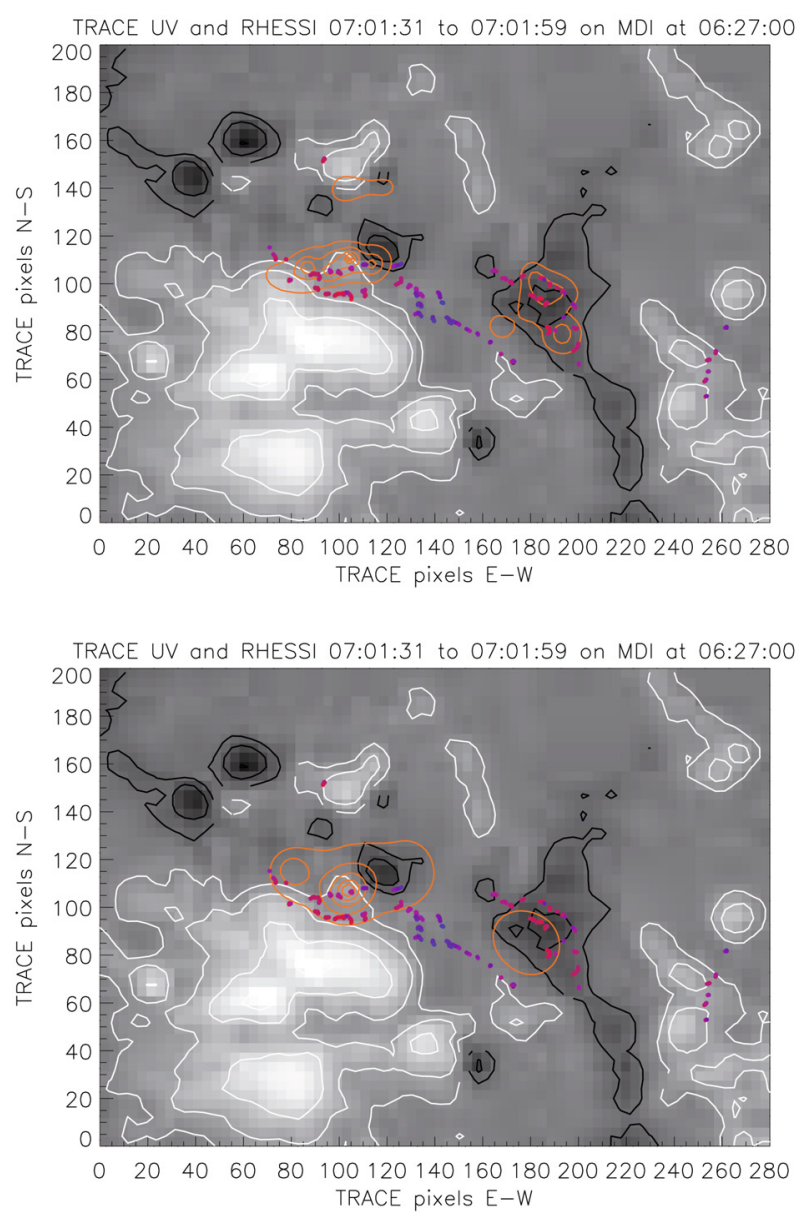

Fig. 12. Colour-coded partial tracks of UV footpoints on an MDI magnetogram, with overlaid RHESSI contours at 25-50 keV (left) and $50-100 \mathrm{keV}($ right). The tracks are colour-coded by flux transfer rate $\dot{\Phi}$, with colour scale blue/purple/dark pink/red. Blue corresponds to $\log _{10}\left(\dot{\Phi} \mathrm{V} \mathrm{m}^{-1}\right)=0.5$ and red to $\log _{10}\left(\dot{\Phi} \mathrm{V} \mathrm{m}^{-1}\right)=4$.

By applying an à trous wavelet filtering algorithm to the lightcurves we have selected bursty features on timescales of the order of $10 \mathrm{~s}$, and performed a linear cross-correlation on these filtered lightcurves. We can identify well-separated pairs of footpoints showing significant correlations, which are likely to reveal conjugate footpoints. In many cases, the correlation time lag is less than one second, as would be expected from particle beams, however in some cases it is on the order of a few seconds. If not a random co-incidence, it may be that such footpoints "communicate" by Alfvén waves. Both the inferred connectivity and changes in connectivity are more complicated than in for example the very well-organised flare analysed by Grigis \& Benz (2005), in which pairs of X-ray footpoints light up in sequence along a neutral line. Our flare takes place in a part of the active region with two major neutral lines, on either side of the main negative polarity and most of the connections reach across one or other of these neutral lines. The "convergence" of UV footpoints towards the location of the strongest X-ray footpoint discussed in Sect. 5 is consistent with footpoints spreading outwards from both of these neutral lines, suggesting reconnection taking place simultaneously at both neutral lines. We have also found several correlated footpoint pairs where both partners are in positive magnetic field regions. The correlated brightening of these distant locations might suggest some large-scale excitation of the field, such as by a wave pulse traveling through the
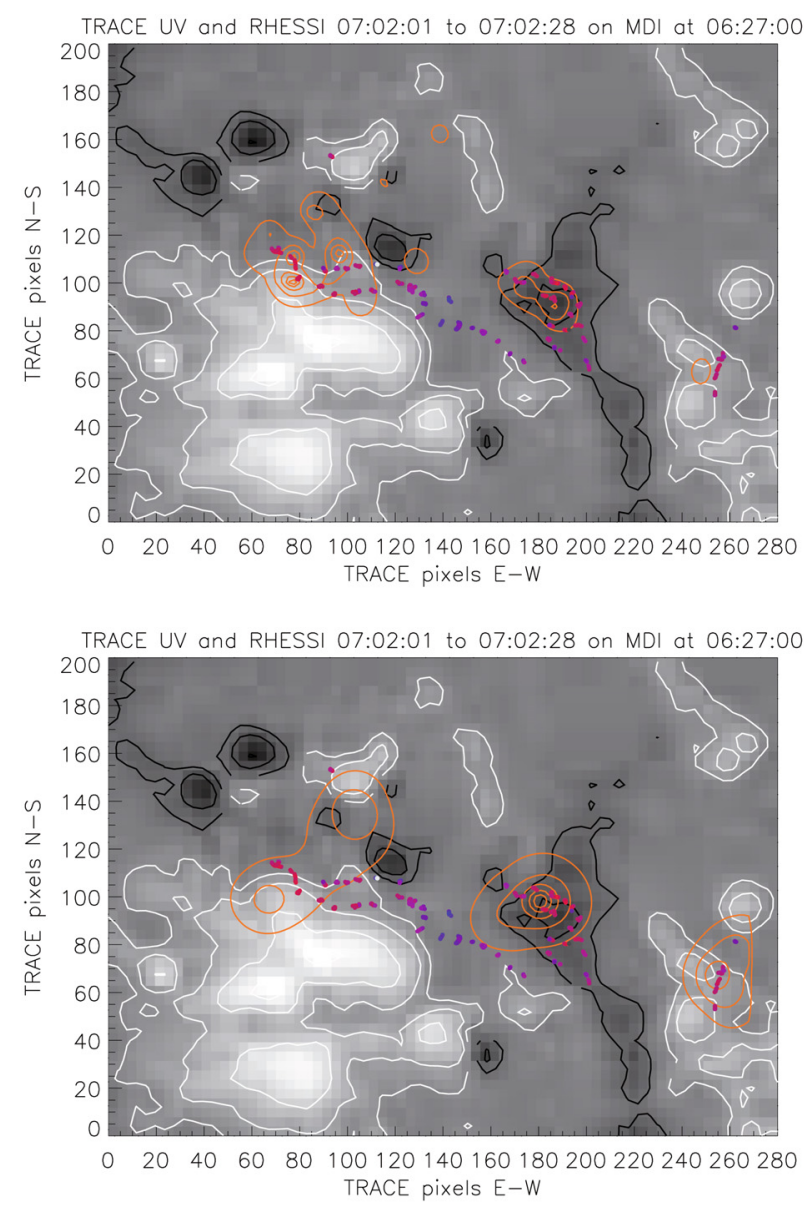

Fig. 13. As Fig. 12 but both $\dot{\Phi}$ and HXR sources shown 30 s later.

region. However it would have to be a large-scale excitation that affected only a small fraction of footpoints in such a way as to give correlated brightenings. This leads us to speculate instead that the positive-to-positive correlations suggest communication via a magnetic null.

Grigis \& Benz (2005) also show that elementary X-ray flare bursts occur as reconnection progresses to different parts of a reconnecting structure. Splitting up the overall TRACE lightcurve in Fig. 6 shows that different UV footpoint groups brighten at different times, consistent with the Grigis \& Benz picture. However it should also be noted that Fletcher et al. (2004) found that the lightcurve of an individual UV footpoints is correlated with the reconnection rate as measured by the the progress of that footpoint, so that if we assume one footpoint is connected to a single reconnection site (as the connectivity analysis indeed assumes) it becomes clear that the overall UV behaviour may be a combination of a varying reconnection rate and a changing reconnection site.

We have also presented a comparison of the location of hard X-ray sources with an observationally-determined value of the flux transfer rate (in 2D this can be interpreted directly as an electric field strength). The HXR sources concentrate at locations where the flux transfer rate is high. The flux transfer rate if interpreted as an electric field is in all locations greatly superDreicer (the Dreicer field being $\sim 0.1 \mathrm{~V} \mathrm{~m}^{-1}$ in the corona). In a $2 \mathrm{D}$ reconnecting configuration with a (non-reconnecting) guide field, some component of this electric field is parallel to the guide field, and particle acceleration in a weakly-magnetised region around the current sheet or X-line is possible. In a 3D geometry 
the flux transfer rate is also related to a parallel electric field. It is doubtful that a single reconnecting structure with dimensions large enough to accelerate sufficient electrons for a flare can exist, particularly in the flare impulsive phase, however it is entirely reasonable that the rate at which the field is reconfiguring, as revealed by the flux transfer rate, has a bearing on the rate at which magnetic free energy is converted to the kinetic energy of fast particles.

It is clear that with this analysis we are pushing the limits of the TRACE and MDI data, both in the time resolution and continuous sequences required to make clean time-correlations, and the spatial resolution required to isolate the individual UV footpoints. But we have nonetheless been able to show the utility of such an approach, and look forward to its application to data from the new generation of solar observatories; Hinode and in the future also SDO.

Acknowledgements. This work was supported by the Nuffield Foundation, by PPARC and STFC under a Rolling Grant and by the European Commission through the SOLAIRE Network (MTRN-CT-2006-035484). I am grateful to Dr. Clare Foullon for suggesting the use of the à trous algorithm in this work, and to the late, and much missed, Dr. Thomas R. Metcalf for assistance with the RHESSI imaging.

\section{References}

Asai, A., Yokoyama, T., Shimojo, M., et al. 2004, ApJ, 611, 557 Aschwanden, M. J., Schwartz, R. A., \& Alt, D. M. 1995, ApJ, 447, 923 Bogachev, S. A., Somov, B. V., Kosugi, T., \& Sakao, T. 2005, ApJ, 630, 561 De Moortel, I., Ireland, J., \& Walsh, R. W. 2000, A\&A, 355, L23
De Pontieu, B., Erdélyi, R., \& de Wijn, A. G. 2003, ApJ, 595, L63

Emslie, A. G., \& Sturrock, P. A. 1982, Sol. Phys., 80, 99

Fletcher, L., \& Hudson, H. S. 2002, Sol. Phys., 210, 307

Fletcher, L., \& Hudson, H. S. 2008, ApJ, 675, 1645

Fletcher, L., Pollock, J. A., \& Potts, H. E. 2004, Sol. Phys., 222, 279

Gary, G. A., \& Moore, R. L. 2004, ApJ, 611, 545

Gorbachev, V. S., Kelner, S. R., Somov, B. V., \& Shvarts, A. S. 1988, AZh, 65, 601

Grigis, P. C., \& Benz, A. O. 2005, ApJ, 625, L143

Harra, L. K., Démoulin, P., Mandrini, C. H., et al. 2005, A\&A, 438, 1099

Hesse, M., Forbes, T. G., \& Birn, J. 2005, ApJ, 631, 1227

King, D. B., Nakariakov, V. M., Deluca, E. E., Golub, L., \& McClements, K. G. 2003, A\&A, 404, L1

Kopp, R. A., \& Pneuman, G. W. 1976, Sol. Phys., 50, 85

Krucker, S., Hurford, G. J., \& Lin, R. P. 2003, ApJ, 595, L103

Lee, J., Gary, D. E., \& Choe, G. S. 2006, ApJ, 647, 638

Lin, R. P., Dennis, B. R., Hurford, G. J., et al. 2002, Sol. Phys., 210, 3

Litvinenko, Y. E., \& Somov, B. V. 1993, Sol. Phys., 146, 127

Maclean, R. C., Hornig, G., Priest, E. R., \& Beveridge, C. 2006, Sol. Phys., 235, 259

Melrose, D. B. 1992, ApJ, 387, 403

Metcalf, T. R., Alexander, D., Hudson, H. S., \& Longcope, D. W. 2003, ApJ, 595,483

Poletto, G., \& Kopp, R. A. 1986, in The Lower Atmosphere of Solar Flares; Proceedings of the Solar Maximum Mission Symposium, ed. D. F. Neidig, 453

Qiu, J., Lee, J., Gary, D. E., \& Wang, H. 2002, ApJ, 565, 1335

Saba, J. L. R., Gaeng, T., \& Tarbell, T. D. 2006, ApJ, 641, 1197

Sakao, T., Kosugi, T., Masuda, S., et al. 1994, in Proceedings of Kofu Symposium, 169

Starck, J.-L., \& Murtagh, F. 2002, Astronomical image and data analysis, ed. J.-L. Starck, \& F. Murtagh (Berlin: Springer)

Starck, J.-L., Siebenmorgen, R., \& Gredel, R. 1997, ApJ, 482, 1011

Temmer, M., Veronig, A. M., Vršnak, B., \& Miklenic, C. 2007, ApJ, 654, 665 